\title{
Estudo comparativo da implementação da Modelagem da Informação da construção em obras públicas no Brasil e no Reino Unido
}

Comparative study of the implementation of building Information Modelling in public works in Brazil and the United Kingdom

Estudio comparativo de la implementación del Modelado de Información de construcción en obra pública en Brasil y Reino Unido

Recebido: 05/01/2021 | Revisado: 05/01/2021 | Aceito: 10/01/2021 | Publicado: 12/01/2021

Daniele Moreira Santos

ORCID: https://orcid.org/0000-0002-9300-7994 Centro Estadual de Educação Tecnológica Paula Souza, Brasil E-mail: daniele.msantos@fco.net.br

Fabrício José Piacente

ORCID: https://orcid.org/0000-0001-8306-4541 Centro Estadual de Educação Tecnológica Paula Souza, Brasil E-mail: fjpiacente@yahoo.com.br

José Manoel Souza das Neves

ORCID: https://orcid.org/0000-0002-7277-9434 Centro Estadual de Educação Tecnológica Paula Souza, Brasil E-mail: jmneves.fatec@gmail.com

Marília Macorin de Azevedo

ORCID: https://orcid.org/0000-0003-0225-8155 Centro Estadual de Educação Tecnológica Paula Souza, Brasil E-mail: marilia.azevedo@fatec.sp.gov.br

\begin{abstract}
Resumo
O escalonamento de custo e cronograma em grandes projetos é um problema histórico observado mundialmente. No Brasil esse problema é recorrente em obras do setor público. Inovações disruptivas como a Modelagem da Informação da Construção ou Building Information Modeling (BIM) surge como solução, porém é apenas uma parte do processo de melhoria e a sua sinergia com outras técnicas como a Construção Enxuta pode resultar em melhorias significativas no desempenho da indústria da construção. Por tanto este artigo tem como objetivo comparar a implementação do BIM e identificar os benefícios alcançados. Para este fim foi realizada uma revisão da literatura somada a uma pesquisa documental com a finalidade de identificar as estratégias implementadas pelo Reino Unido e o Brasil bem como os benefícios alcançados.
\end{abstract}

Palavras-chave: Modelagem da informação da construção; Lean construction; Lean-BIM.

\begin{abstract}
Cost and schedule escalation in large projects are a historical problem observed worldwide. In Brazil, this problem is recurrent in public sector works. Disruptive innovations such as Building Information Modeling (BIM) come as a solution, but it is only part of the improvement process and their synergy with other techniques such as Lean Construction can result in significant improvements in the construction industry's performance. Therefore, this article aims to compare the implementation of BIM and identify the benefits achieved. To this end, a literature review was added to a documentary research to identify the strategies implemented by the United Kingdom and Brazil as well as the benefits achieved.
\end{abstract}

Keywords: Building information modelling; Lean construction; Lean-BIM.

\section{Resumen}

El escalado de costos y cronogramas en proyectos grandes es un problema histórico observado en todo el mundo. En Brasil, este problema es recurrente en las obras del sector público. Las innovaciones disruptivas como el Modelado de Información de Construcción o Building Information Modeling (BIM) aparecen como una solución, pero es solo una parte del proceso de mejora y su sinergia con otras técnicas como Lean Construction puede resultar en mejoras significativas en el desempeño de la industria de la construcción. Por tanto, este artículo tiene como objetivo comparar la implementación de BIM e identificar los beneficios conseguidos. Para ello, se realizó una revisión de la literatura, además de una investigación documental, con el fin de identificar las estrategias implementadas por Reino Unido y Brasil, así como los beneficios alcanzados.

Palabras clave: Modelado de información de construcción; Lean construction; Lean-BIM. 


\section{Introdução}

Historicamente, grandes projetos de construção sofrem com o escalonamento de custo e cronograma, problema este observado mundialmente. Em muitos dos casos o custo final do projeto se torna maior do que o custo estimado e liberado durante o planejamento inicial, engenharia preliminar, projeto final ou mesmo no início da construção. (Shane et al., 2009)

No Brasil o problema é recorrente nas obras públicas o que torna a temática objeto de análise de órgãos fiscalizadores como o Tribunal de Contas da União (TCU). Outro problema recorrente no Brasil são as obras inacabadas. O TCU realizou um levantamento no qual foram identificadas 400 obras inacabadas das quais 130, de responsabilidade da União, somavam o valor de $\mathrm{R} \$$ 3,5 bilhões. As demais 270 eram gerenciadas pelos estados e municípios, mediante recursos federais. (Tribunal de Contas da União, 2018)

Algumas das causas que contribuem para a paralisação das obras são: o fluxo orçamentário e financeiro, problemas no projeto e execução da obra. (Tribunal de Contas da União, 2006) Estas causas são reflexos da adoção de um processo falho durante as licitações, pois muitas dessas são elaboradas com base em projetos incompletos.

O Building Information Modelling (BIM) ou Modelagem da Informação da Construção surge como solução para os problemas da indústria da construção. Por meio do BIM os processos de construção se tornam cada vez mais automatizados, com projetos em 3D, modelagem de custos e gerenciamento de instalações. Com isso a implementação do BIM em projetos de construção ganhou força e impulso em muitas partes do mundo. (Smith, 2014)

A pesquisa internacional da Mcgraw Hill Construction (2014) constatou que, embora o uso do BIM na indústria brasileira seja algo relativamente novo, nos últimos anos vem ganhando força. Maior país e economia da América Latina a adoção do BIM na construção apresentou um crescimento significativo nos anos de 2014 e 2016. Este movimento foi observado devido a hospedagem de eventos como a Copa do Mundo da FIFA e dos Jogos Olímpicos (Smith, 2014)

No entanto esse crescimento representa a adoção pelas grandes construtoras brasileiras, enquanto no setor público esse crescimento foi inexpressivo. A fim de reverter o cenário da indústria da construção brasileira e seguindo a tendência mundial o governo brasileiro lançou no dia 16 de maio de 2018 a estratégia BIM BR que foi instituída pelo Decreto $\mathrm{n}^{\circ}$ 9.377, de 17 de maio de 2018, com a finalidade de promover a inovação na indústria da construção. (Ministério da Indústria Comércio Exterior e Serviços, 2018)

Porém a implementação desse novo conceito e processo organizacional requer uma mudança profunda de âmbito cultural da indústria da construção. Um conceito que pode auxiliar na agilidade desse processo de transição é o Lean Construction como foi observado no Reino Unido. (Dave et al., 2013)

Portanto este trabalho tem como objetivo comparar a implementação do BIM e identificar os benefícios alcançado. Para esse fim foi realizada uma revisão da literatura somada a uma pesquisa documental para identificar as estratégias implementadas pelo Reino Unido e o Brasil.

\section{Fundamentos Teóricos}

\subsection{Building Information Modelling}

Historicamente é possível identificar vestígios do início do Building Information Modelling (BIM), em 1982 com o desenvolvimento do programa ArchiCAD, mas foi em 2000, com o desenvolvimento do programa Revit, que se observou um movimento em direção à sua implementação. (Smith, 2014b)

Erroneamente definido como um sistema ou ferramenta de modelação tridimensional, o BIM é na realidade um conceito de integração paramétrica de objetos alimentado por um repositório de informações que facilita a interoperabilidade e o intercâmbio de informações com programas relacionados. (Miettinen; Paavola, 2014)

Mettinen e Paavola (2014) afirmam que o uso colaborativo reduz erros de projeto e aumenta a produtividade da 
indústria da construção estabelecendo assim um novo padrão emergente para o gerenciamento da construção bem como uma mudança tecnológica e processual no setor da construção civil. O que logo o torna um conceito a ser adotada para garantir que ocorra a integração da informação e a mudança do padrão documental para o padrão banco de dados integrado. (Bryde; Broquetas; Volm, 2013)

Com evolução constante o BIM apresenta fases que são descritas em termos de dimensões. Eastman et al. (2008) definiram essa capacidade multidimensional como modelagem "nD", pois ela tem a capacidade de adicionar um número quase infinito de dimensões ao modelo de construção. Atualmente as dimensões existentes são: 3D (modelo do objeto), 4D (tempo), 5D (custo), 6D (operação), 7D (sustentabilidade) e 8D (segurança). (Smith, 2014)

Esta capacidade multidimensional exigi dos profissionais uma adaptação rápida para fornecer serviços mais sofisticados que incorporem principalmente o 4D planejamento, 5D modelagem de custos e gerenciamento de instalações e por fim 6D compartilhamento de informações e dados de custos. (Smith, 2014)

Segundo McGraw Hill (2014) está mudança vem varrendo o mundo e as equipes de projeto são beneficiadas pela rápida comunicação, computadores mais potentes e móveis, modelagem digital robusta e uma mudança transformadora em direção a processos de entrega integrados, todos gerando resultados positivos, eficientes e benefícios inimagináveis.

Assim a implementação do BIM em projetos de construção está ganhando força e impulso em muitos países ao redor do mundo. (Smith, 2014) Dentre estes países Hore, McAuley e West (2017) destaca os resultados do Reino Unido que alcançou a eficiência no maior número de componentes da maturidade. Decorrência de uma estratégia de implementação que objetivou não somente a adoção do BIM, mas também a de outras técnicas como o Lean Construction.

\subsection{Lean Construction}

Para melhor compreensão do conceito Lean Construction (LC) ou Construção Enxuta, primeiramente é necessário entender o conceito originário Lean. Com base no Sistema Toyota de Produção (STP) o termo Lean Manufacturing ou indústria enxuta, foi mencionado pela primeira vez em um estudo da indústria automobilística japonesa Toyota com críticas a indústria automobilística americana que utilizava um sistema de produção em massa. (Womack, Jones \& Roos, 2004)

A necessidade da adoção do conceito Lean na construção civil é defendida por Koskela (1992) que por meio de um estudo afirmou que a base conceitual de gerenciamento e engenharia de construção, baseada apenas no conceito de conversão ${ }^{1}$, é obsoleta. Ou seja, a construção deve ser vista como processos de fluxo que consistem em atividades de desperdício e conversão.

Estes processos de fluxo devem ser projetados, controlados e aprimorados na prática constantemente e para auxiliar nessa tarefa foram elaborados por Koskela (1992) 11 princípios. São eles: redução do compartilhamento de atividades que não agregam valor; aumento do valor de saída por meio de consideração sistemática do cliente; redução da variabilidade; redução do tempo do ciclo; simplificação do número de etapas, peças e ligações; aumento da flexibilidade da saída; aumento da transparência do processo; foco no controle de todo o processo; construção contínua de melhoria do processo; equilíbrio na melhoria do fluxo versos a melhoria da conversão e prática do benchmark.

Ainda segundo Koskela (1992) os esforços de desenvolvimento da construção, como industrialização, construção integrada por computador e automação da construção, precisam ser redefinidos de forma a atender a necessidade de equilibrar a melhoria do fluxo e da conversão.

uanto da implementação, o processo é dividido em três fases sobrepostas.: estágio 1 , fase de transição em que o foco

\footnotetext{
${ }^{1}$ Modelo conceitual dominante na construção civil é conceito conversão definido como um conjunto de atividades que transformam os insumos (materiais, informação) em produtos intermediários.
} 
está em tornar a organização mais enxuta; Estágio 2, fase em que se concentra no alcance do estado Lean como um ponto final; e o estágio 3, fase que foca no processo de transformação da gerência, para que ela permaneça enxuta e responsiva. (Green; May, 2005)

Contudo, Sacks (2010) e Tomioko e Neves (2019) apontaram que para alcançar a total eficiência do Lean Construction é necessária sua adoção integrada a outras técnicas.

\subsection{Building Information Modelling e Lean Construction}

O Building Information Modelling e a Lean Construction (LC) existem como dois conceitos diferentes com o objetivo de implementar melhorarias a indústria da construção, com comunidades e interesses distintos. Nos últimos 10 anos, ambos começaram a se difundir separadamente na prática avançada, com velocidade acelerada pelo mundo.

No entanto recentemente um discernimento de que esses dois conceitos têm uma sinergia mútua considerável e que é altamente vantajoso implementá-las em conjunto ganhou força, primeiro entre os profissionais pioneiros e depois entre os acadêmicos. (Dave et al., 2013)

Entre esses acadêmicos temos Rischmoller, Alarcón e Koskela (2006) que por meio de um quadro teórico baseado em um conjunto dos princípios Lean tentam demonstrar o impacto do que eles denominaram "Computer Advanced Visualization Tools" (CAVTs). Em outro esforço para integrar os processos LC ao BIM, Khanzode et al. (2006) tentou montar uma estrutura conceitual para vincular o Virtual Design \& Construction (VDC) ao Lean Project Delivery Process (LPDS).

Sacks et al. (2009) por meio de uma revisão da literatura e criação de uma estrutura taxonomia de análises avaliaram as interconexões do LC e do BIM. Mas o estudo de maior impacto foi o realizado por Sacks et al. (2010) que identificaram 56 interações demonstrando assim as sinergias positivas e negativas entre o Lean e o BIM.

Contudo o conceito de sinergia entre o Lean e o BIM ganhou força quando o governo do Reino Unido (UK) reconheceu esse vínculo e declarou sua intenção de desenvolver processos do LC para aproveitar o trabalho anterior do BIM Task Group's, grupo criado para difundir o conceito BIM no UK. (Dave et al., 2013)

Em vista disso surgi a necessidade de conscientizar os usuários do BIM sobre os princípios, métodos, ferramentas e benefícios do Lean, bem como as empresas implementadoras do conceito Lean sobre as funcionalidades do BIM. Dave et al. (2013) destacam em seu estudo quatro principais maneiras de como o LC e o BIM interagem de forma benéfica.

São elas: 1. BIM contribui diretamente para os objetivos LC; 2. O BIM permite processos LC e contribui indiretamente para os objetivos LC; 3. Os sistemas de informações auxiliares, habilitados pelo BIM, contribuem direta e indiretamente para o alcance das metas do LC; e 4. Os processos LC facilitam a introdução do BIM. (Dave et al., 2013) Logo fica evidente que essa sinergia é benéfica e principalmente necessária no que referisse a implementação seja do LC ou do BIM na indústria da construção.

\section{Metodologia}

A elaboração desse artigo contempla uma combinação de métodos. A pesquisa é classificada como qualitativa e apresenta como característica principal o cunho exploratório.

O primeiro método aplicado foi a análise bibliométrica. Por meio dela foi possível identificar os autores que tem se dedicado a estuda a sinergia Lean/BIM, Figura 1. Outro resultado observado por meio da bibliometria foi a aderença do tema no cenário mundial. Para a elaboração do referencial teórico foi empregada a revisão literária para descrever o conhecimento científico atual sobre o tema. 
Figura 1 - Fluxograma do desenvolvimento da pesquisa.

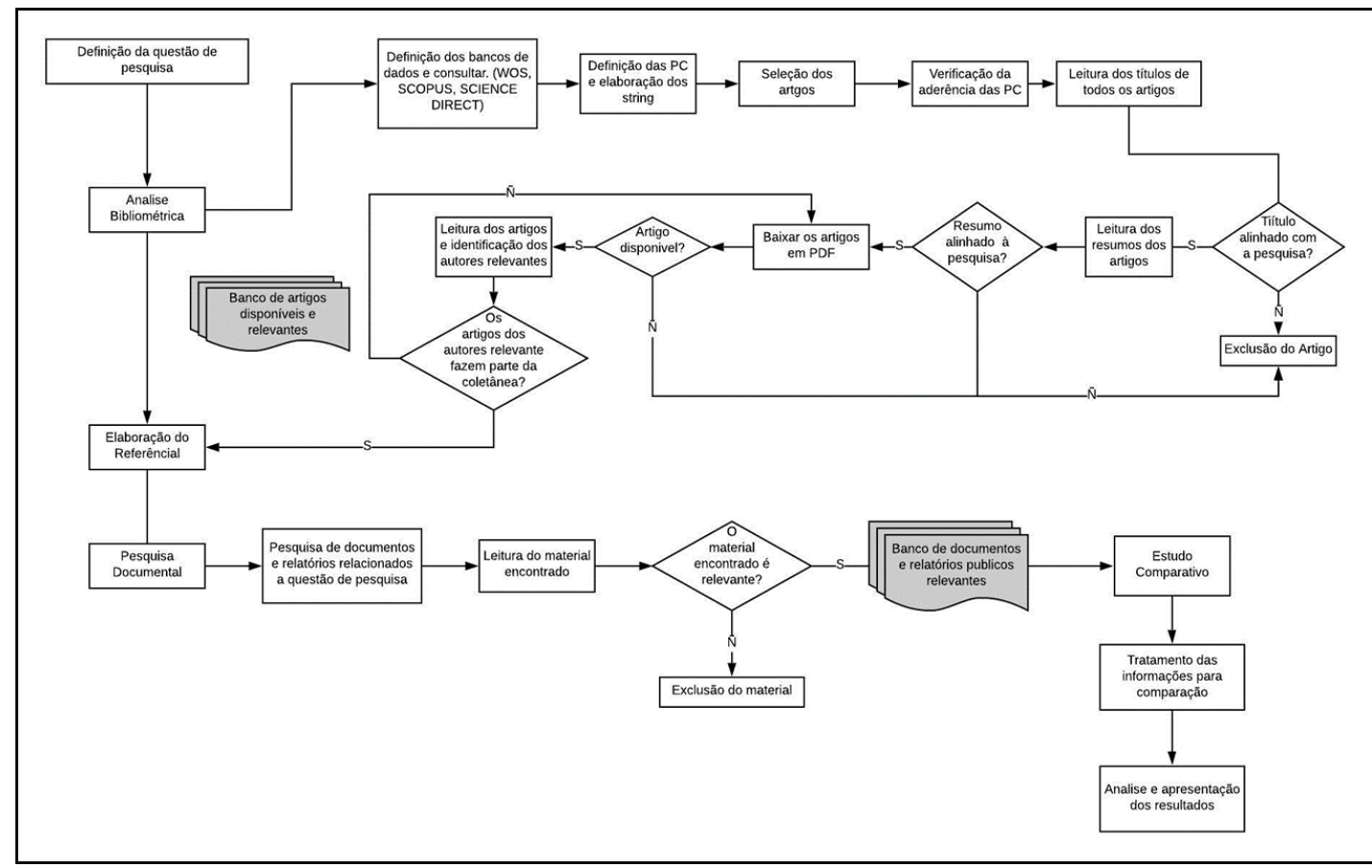

Fonte: Autores.

Para a próxima etapa desse artigo foram aplicados mais três métodos. A pesquisa documental, cujo materiais pesquisados foram relatórios públicos, procedimentos, e projetos disponíveis digitalmente pelos governos do Brasil e do Reino Unido.

Para organizar as informações obtidas por meio da pesquisa documental utilizou a metodologia: mapas conceituais. Os mapas conceituais de acordo com Pereira A. S. et. al. (2018) auxilia na organização de grandes quantidades de informações ou conceitos que possuem relação entre si. Essa organização facilita a compreensão das informações que se pretende passar ou aprendizado de um conceito.

Nessa pesquisa o mapa conceitual foi aplicado para ambas as finalidades. Primeiramente para sintetizar o conceito da metodologia BIM e apresentar a cronologia evolutiva da implementação do método. Posteriormente utilizou-se de mapas conceituais para organizar as informações resultantes da pesquisa documental. Assim tornou-se possível realizar a análise comparativa da cronologia de implementação da metodologia BIM entre Brasil e Reino Unido.

O estudo comparativo foi a último método a ser aplicado. Definido por Bulgacov (1998) como a comparação sistemática de um grupo determinado ou específico com finalidade de estabelecer relações entre suas variáveis ou categorias analíticas. A aplicação do método se mostrou apropriado para responder à questão de pesquisa.

\section{Resultados e Discussão}

Os resultados apresentados são oriundos da comparação das estratégias de implementação do BIM adotadas pelo Reino Unido (UK) e o Brasil. Ao longo da revisão bibliográfica foi constado que o UK criou uma estratégia que incorporou diferentes conceitos que se complementam com o objetivo de informatizar a indústria da construção civil e assim alcançar os objetivos por eles estabelecidos.

A estratégia do UK se mostrou eficiente apresentando bons resultados. O que tomou o UK líder em fornecer padrões e descrições do BIM a outros países que o estão usando como modelo para melhores práticas em BIM. (National Building Specification, 2016) 
O Brasil foi um desses países que reconheceu o sucesso da experiencia da UK na implementação do BIM. De forma que o Ministério da Indústria, Comércio Exterior e Serviços da República Federativa do Brasil (MDIC) assinou em dezembro de 2016 o Memorando de Entendimento (MOU) com o secretário de Estado do Comércio Internacional, Dr. Liam Fox, concordando com o intercâmbio de informações sobre estratégias nacionais para disseminação do BIM. (HM Government, 2016)

Apesar de cada país definir a sua estratégia com base nos seus objetivos, contexto político e econômico, ainda assim é possível identificar semelhanças. Nos dois casos estudados, Quadro 1, observa-se que a estrutura de adoção e disseminação são parecidas nos dois países. Ambos optaram por uma implementação de nível nacional por meio de decreto com apoio, divulgação e liderança governamental.

Quadro 1- Comparativo das estratégias de implementação do Building Information Modelling.

\begin{tabular}{|c|c|c|}
\hline & Brasil & Reino Unido \\
\hline Política & Adoção por meio de decreto. & Adoção por meio de decreto. \\
\hline Apoio político & Central & Central \\
\hline Normas & Nacional & Nacional + Departamental \\
\hline Direcionamento & Nacional & Nacional \\
\hline Nível de Maturidade & Nível $1 \rightarrow$ Nível 2 & Nível 2 $\rightarrow$ Nível 3 \\
\hline Estratégia & BIM BR & UK BIM Task Group \\
\hline Objetivos & $\begin{array}{l}\text { Assegurar ganhos de produtividade } \\
\text { ao setor de construção civil; } \\
\text { Proporcionar ganhos de qualidade } \\
\text { nas obras públicas; } \\
\text { Aumentar a acurácia no planejamento } \\
\text { de execução de obras proporcionando } \\
\text { maior confiabilidade de cronogramas } \\
\text { e orçamentação; } \\
\text { Contribuir com ganhos em } \\
\text { sustentabilidade por meio da redução } \\
\text { de resíduos sólidos da construção } \\
\text { civil; } \\
\text { Reduzir prazos para conclusão } \\
\text { de obras; } \\
\text { Contribuir com a melhoria da } \\
\text { transparência nos } \\
\text { licitatórios; } \\
\text { Reduzir necessidade de aditivos } \\
\text { contratuais de alteração do projeto, de } \\
\text { elevação de valor e de prorrogação de } \\
\text { prazo de conclusão e de entrega da } \\
\text { obra; } \\
\text { Elevar o nível de qualificação } \\
\text { profissional na atividade produtiva; }\end{array}$ & $\begin{array}{l}\text { Alinhar o projeto e a construção à gestão dos } \\
\text { custos operacionais baseadas em resultados } \\
\text { versus critérios claros de desempenho; } \\
\text { Criar um alinhamento de interesse entre } \\
\text { aqueles que projetam/constroem e aqueles que } \\
\text { posteriormente o ocupam e operam; } \\
\text { Aplicação de padrões comuns; } \\
\text { Controle do projeto e incentivo à redução de } \\
\text { custos; } \\
\text { Incentivar o uso do benchmarking e feedback } \\
\text { de projetos anteriores para constante melhoria; } \\
\text { Estabelecer um preço de mercado competitivo } \\
\text { para que o custo se torne um fator importante } \\
\text { na concepção e entrega do projeto; } \\
\text { Melhorar a transparência por meio de dados } \\
\text { completos e acessíveis; } \\
\text { Eliminar o desperdício, simplificando os } \\
\text { processos; } \\
\text { Explorar modelos alternativos de compras que } \\
\text { introduzam competitividade, }\end{array}$ \\
\hline
\end{tabular}




\begin{tabular}{|c|c|c|}
\hline & $\begin{array}{l}\text { Estimular a redução de custos } \\
\text { existentes no ciclo de vida dos } \\
\text { empreendimentos. }\end{array}$ & \\
\hline Metas & $\begin{array}{l}\text { Aumentar a produtividade das } \\
\text { empresas em 10\%; } \\
\text { Reduzir custos em 9;7\%; } \\
\text { Aumentar em } 10 \text { vezes a adoção do } \\
\text { BIM ( } \% \text { do PIB da construção civil); } \\
\text { Elevar em } 28,9 \% \text { o PIB da } \\
\text { construção civil. }\end{array}$ & $\begin{array}{l}\text { Redução de } 33 \% \text { no custo inicial de construção } \\
\text { e no custo de toda a vida útil dos ativos } \\
\text { construídos; } \\
\text { Redução de } 50 \% \text { no tempo total, desde o início } \\
\text { até a conclusão, para ativos de nova construção } \\
\text { e reformados; } \\
\text { Redução de } 50 \% \text { nas emissões de gases de } \\
\text { efeito estufa no ambiente construído; } \\
\text { Redução de } 50 \% \text { na diferença comercial entre o } \\
\text { total de exportações e o total de importações de } \\
\text { produtos e materiais de construção. }\end{array}$ \\
\hline
\end{tabular}

Fonte: Ministério da Industria, Comércio Exterior e Serviços (2018), HM Government (2011a), Fenby-taylor et al. (2016).

As diferenças se concentram nas metas estabelecidas por cada um dos governos. As metas brasileiras são claramente direcionadas ao setor econômico, pois foca no crescimento do Produto Interno Bruto (PIB) por meio do aumento da produtividade da indústria da construção. Já as metas do Reino Unido são pulverizadas entre o crescimento econômico, qualidade da construção e sustentabilidade.

Apesar do Reino Unido se encontrar atualmente no nível 2 de maturidade e está trabalhando para alcançar o nível 3 até 2025, na época do lançamento da sua estratégia de implementação, ele se encontrava no nível 1, Figura 2, assim com o Brasil. Para alcançar três das suas principais metas o Brasil precisa atingir o nível 2 de maturidade, pois somente nessa fase é possível executar as dimensões 4D (tempo) e 5D (custo), como explica Smith (2014).

Figura 2 - Níveis de maturidade do Building Information Modelling.

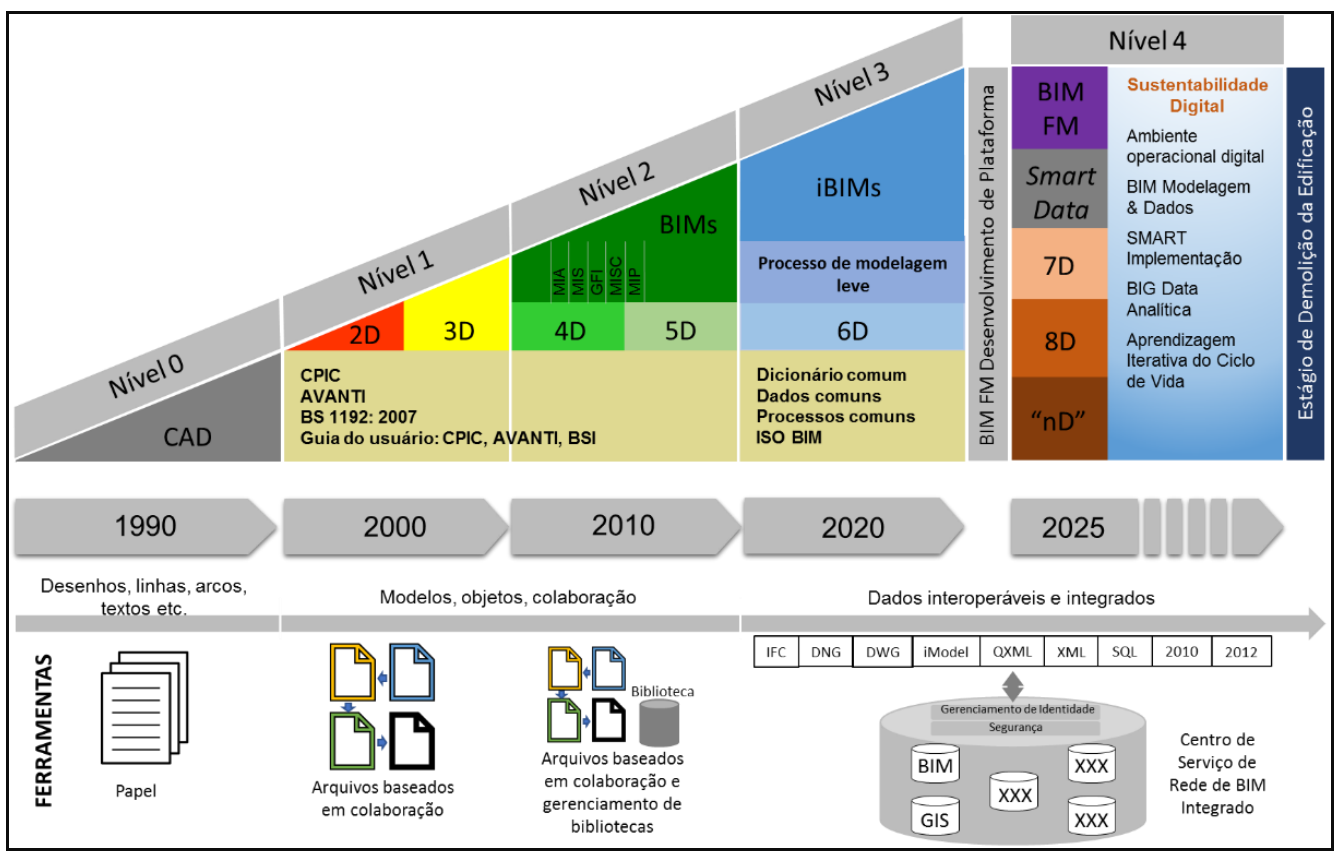

Fonte: HM Government (2011a). 
Estas dimensões são fundamentais para um bom planejamento e controle de custo. Ao atingir esse nível será possível ao Brasil reduzir o número de obras paralisadas ou inacabadas como relatado pelo Tribunal de Contas da União (2018). Pois os motivos apontados como causas que contribuem para a paralisação das obras como, projetos incompletos, fluxo orçamentário e financeiro, são minimizados pelo uso das dimensões 3D (modelagem), 4D (tempo) e 5D (custo).

Em uma análise crítica da jornada de implementação do BIM no Reino Unido, Figura 3, se observa elementos relevantes que favoreceram a sua rápida adoção pela indústria da construção. O UK BIM Task Group foi formado no final de 2011 dando início a mobilização do governo para disseminação do BIM, porém o governo já vinha incentivando outras políticas como a redução de emissão de carbono na construção e a utilização do conceito Lean, sendo este último um diferencial de valor agregador na estratégia UK BIM Task Group.

Figura 3 - Cronologia da implementação do BIM no Reino Unido.

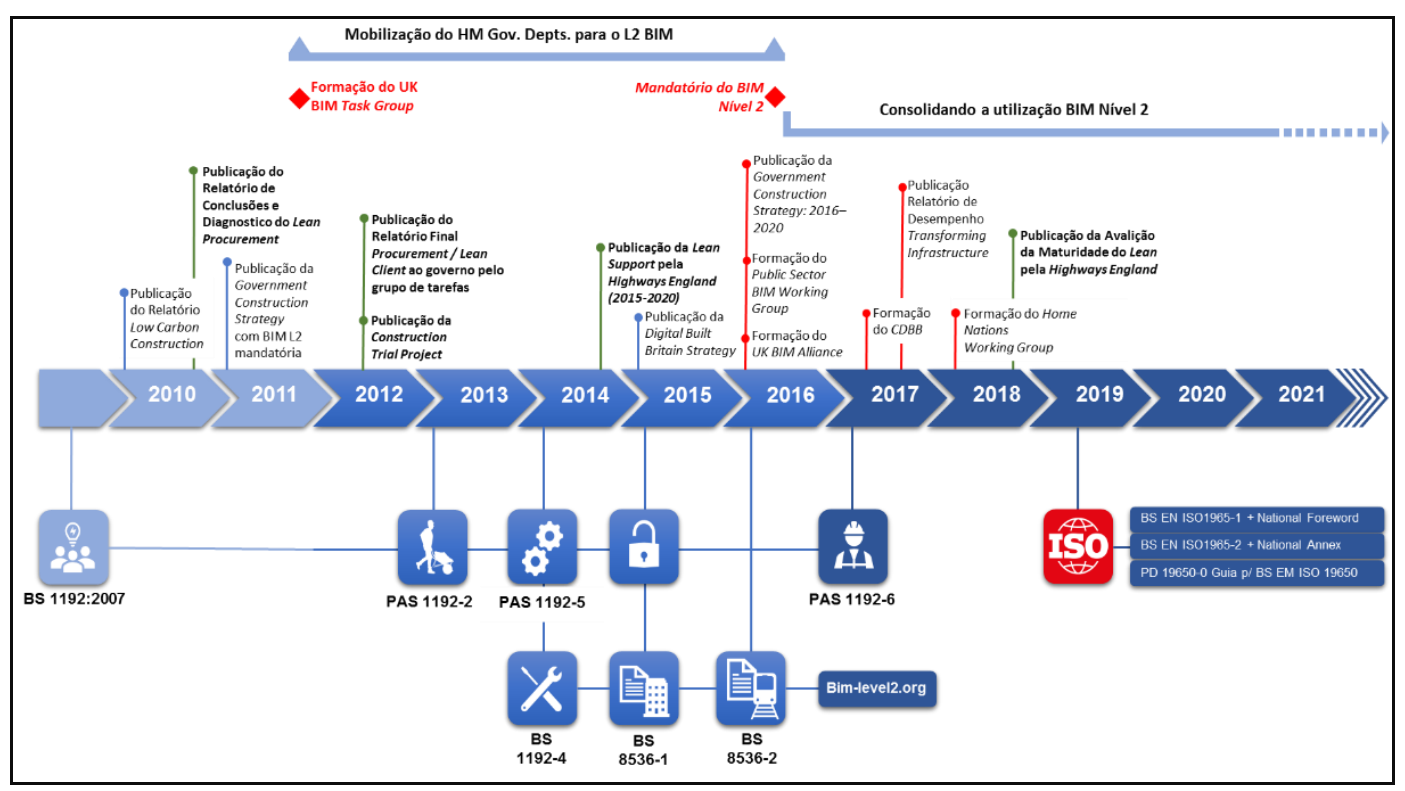

Fonte: Moore (2018), Center for Digital Built Britain (2018).

Como parte de um plano conjunto de melhorarias do desempenho da indústria da construção em termos de custo, valor e emissão de carbono, a estratégia UK BIM foi publicada em março de 2011 apresentando sugestões e recomendações para aumentar a aceitação do BIM ao longo de um período de 5 anos.

No relatório A Report for The Government Construction Client Group o UK BIM Task Group enfatiza que o BIM pode melhorar significativamente a produtividade da construção, no entanto ele é apenas uma parte do processo e como recomendação é sugerido a realização de uma avaliação da sinergia deste com outras técnicas. Uma destas técnicas é o Lean que abordada em conjunto resultaria em melhorias significativas no desempenho da indústria. (HM Government, 2011b)

A etapa de mobilização do governo do Reino Unido durou 4 anos (2012-2016). Logo no início de 2012 é publicado o Construction Trial Project, este documento diferente da estratégia BIM, apresenta as políticas a serem adotadas pela indústria da construção nos anos seguintes a fim de alcançar melhorias na produtividade. Este relatório deixa clara a intenção do governo de acatar a sugestão que o UK BIM Task Group fez no A Report for The Government Construction Client Group de março de 2011.

Ao longo da jornada de implementação outros relatórios focados na disseminação do conceito Lean na construção são publicados pelo governo, são eles: o relatório final Procurement/Lean Client ao governo pelo Task Goup e o Lean Support pela Highways England, este último apresenta a estratégia de adoção do Lean para o período de 2015 a 2020. 
A adoção do BIM em projetos no Reino Unido passou a ser obrigatório no início de 2016. Com isso é formada a UK BIM Alliance com o objetivo de garantir que o BIM passe a ser de uso habitual, transformando e protegendo o futuro da indústria e o Centre for Digital Built Britain (CDBB), órgão financiado pelo governo em parceria com a Universidade de Cambridge, cujo objetivo é apoiar a transformação do setor de construção.

Apesar do acordo assinado entre Brasil e Reino Unido para intercâmbio de informações com o intuito de incentivar a adoção do BIM e das semelhanças entre as estratégias, como por exemplo a instauração de uma data para adoção do BIM por meio de decreto, a jornada de implementação brasileira tem se mostrado diferente, Figura 4.

Figura 4 - Cronologia da implementação do BIM no Brasil.

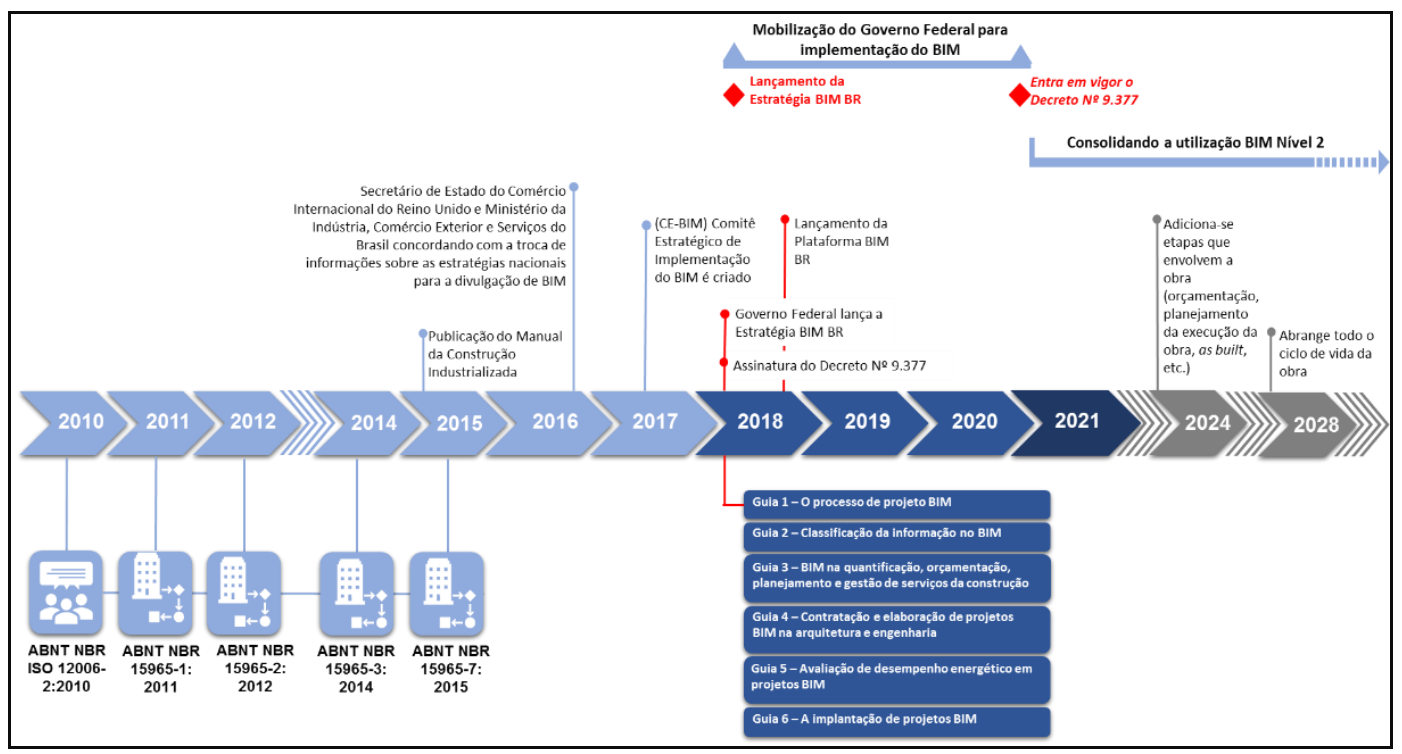

Fonte: Ministério da Industria Comércio Exterior e Serviços (2018), Encontro Técnico Nacional de Auditoria de Obras Públicas (2019).

O início da mobilização do governo federal para implementação do BIM é marcado pela assinatura do decreto n ${ }^{\circ} .377$ em maio de 2018 e relançada no dia 22 de agosto de 2019. Nele o governo dispõe sobre a estratégia nacional de adoção e disseminação do BIM apresentando os principais objetivos e as obrigações do Comitê Gestor da Estratégia do BIM BR.

Anterior a essa data a única iniciativa do governo de promoção da industrialização da construção se deu por meio da publicação do Manual da Construção Industrializada no ano de 2015. Porém sem uma estratégia de divulgação e disseminação não apresentou resultados expressivos no setor.

Um conjunto de normas foram criadas pela Associação Brasileira de Normas Técnicas (ABNT), entre os anos de 2010 e 2015, direcionadas a utilização do BIM. O conjunto de normas definem o sistema de classificação da informação da construção.

Considerando que a adoção compulsória do BIM nos processos licitatórios entra em vigor em janeiro de 2021 e o início da estratégia de disseminação do BIM teve início no primeiro trimestre de 2018, o Brasil almeja alcançar o nível $2 \mathrm{em}$ um período de 3 anos.

Projetos pilotos estão sendo desenvolvido por departamento pré-selecionados na estratégia BIM BR, o Projeto Proarte do Departamento Nacional de Infraestrutura de Transportes (DNIT), Projetos OPUS do Exército Brasileiro e o Projeto Aeroportos da Secretaria de Aviação Civil (SAC). Outros setores públicos apesar de não serem mencionados no BIM BR, também estão investindo na elaborando de projetos pilotos.

Mas diferente do que ocorreu no Reino Unido, onde os projetos pilotos foram executados antes da data decretada pelo 
governo, no Brasil os projetos dos programas pilotos serão adotados para execução somente a partir de janeiro de 2021, data em que entra em vigor o decreto.

Com isso resultados mais contundentes quanto a redução de prazos para conclusão de obras, melhoria da transparência nos processos licitatórios e redução de aditivos contratuais devido a alteração do projeto, elevação de valor e de prorrogação de prazo de conclusão e de entrega da obra, objetivos esperados pelo governo, só poderão ser observados a partir de 2022.

Em contrapartida o sucesso da estratégia UK BIM Task Group pode ser observado que ao logo dos anos, Figura 5. Logo no primeiro ano da estratégia se observou um crescimento de $18 \%$ na utilização do BIM. No decorrer dos anos esse crescimento se mostrou constante chegando à marca de mais de 50 \% de adoção do BIM no ano de 2016.

Figura 5 - Evolução da adoção do BIM no Reino Unido.

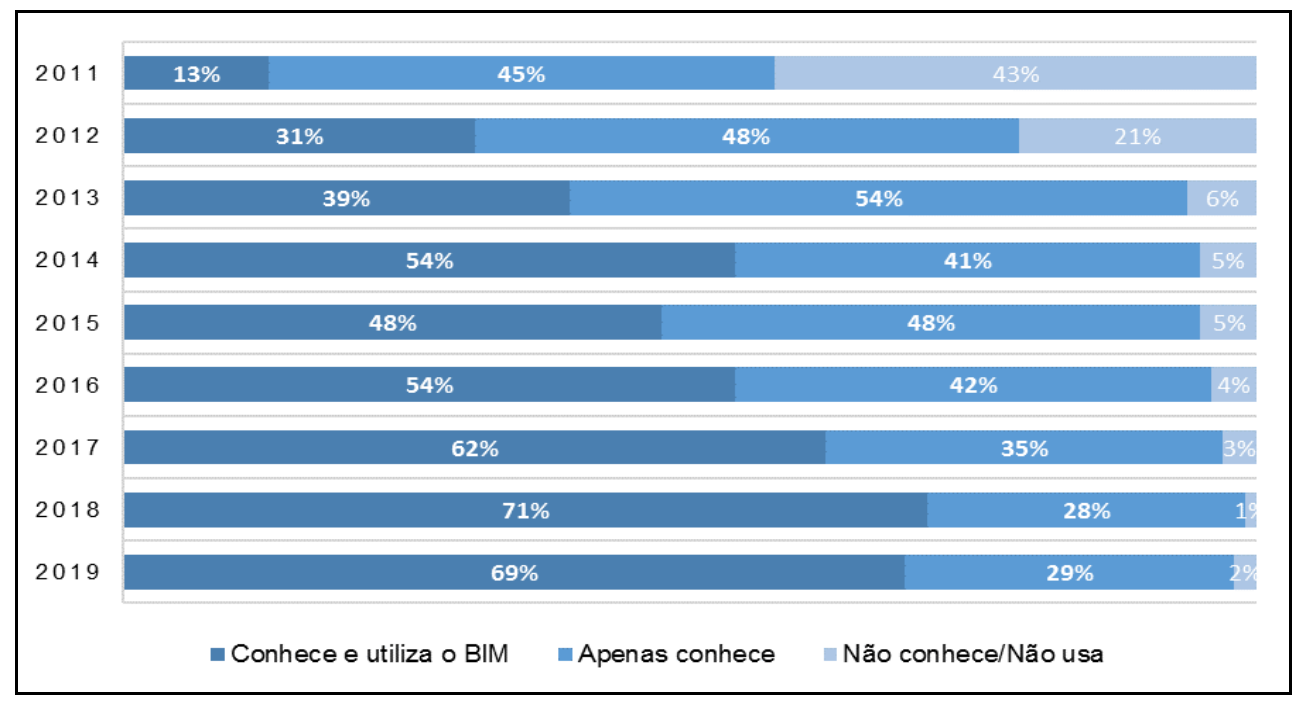

Fonte: National Building Specification (2019).

Apesar do governo britânico ter estabelecido por meio de mandatória a adoção do BIM somente a partir do ano de 2016, por meio de publicações como Goverment Construction Strategy de 2011 e principalmente pelo Construction Trial Project de 2012, ele incentivou a adoção precoce da metodologia. O resultado desse incentivo é observado quando em 2012 cerca de 31\% do público-alvo afirmou conhecer ou utilizar o BIM e 48\% apenas conhecer. Ou seja, em 2012 no Reino Unido, 79\% do público-alvo já tinham tido algum contato com a metodologia BIM. O fato dele não ser algo totalmente novo ou desconhecido do público-alvo viabilizou uma adoção gradativa, porém consistente do BIM. Isso pode ser observado nos resultados dos anos subsequentes.

Analisando esses resultados com base na Curva de Inovação de Rogers, Figura 6, verifica-se que a adoção do BIM já atingiu a massa crítica, ou seja, o número de usuário garante que o conceito BIM seja autossustentável no UK. 
Figura 6 - Curva de adoção de Rogers.

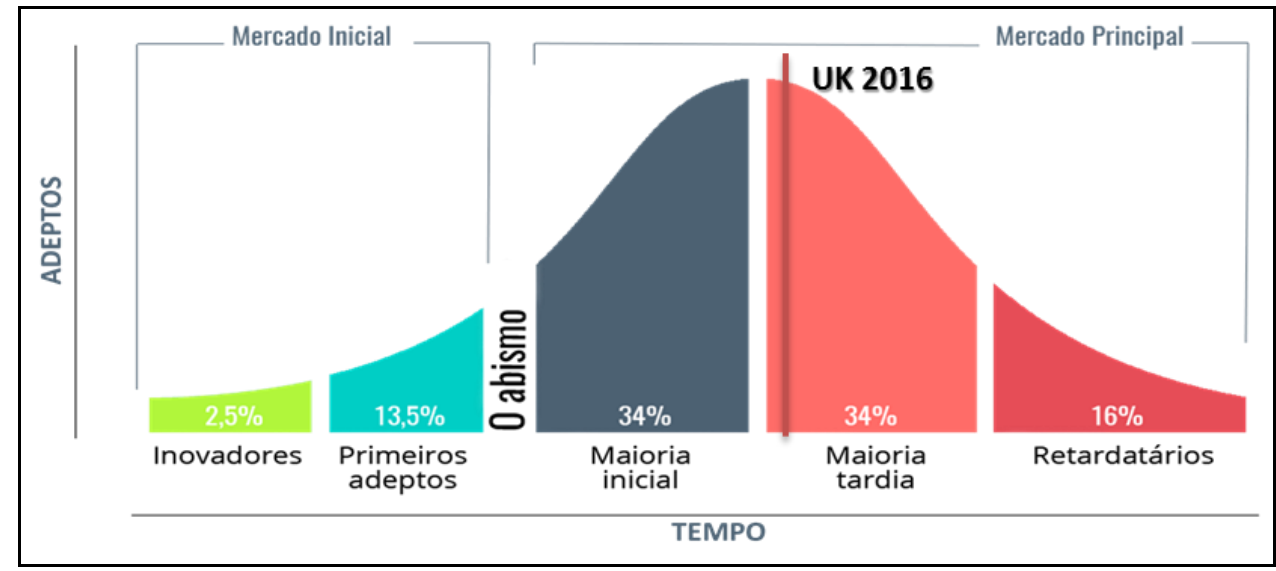

Fonte: National Building Specification (2015).

$\mathrm{Na}$ Curva de Inovação de Rogers representa a evolução da adoção de uma inovação. Em um primeiro momento a inovação é de conhecimento e domínio apenas dos inovadores. Quando a inovação é divulga esperasse uma pequena porcentagem de adeptos. Nesse momento a adoção de uma inovação passa a ter um obstáculo, o que Rogers intitula de 'O Abismo'. O abismo é a resistência ou dificuldade que a inovação tem em ser adotada. Se a inovação conseguir ultrapassar essa barreira ela atinge a massa crítica. A massa crítica é atingida no momento seguinte ao qual se alcança a marca do $50 \%$ de adeptos a inovação. No ano de 2016 a UK apresentou 54\% de adeptos para utilização do BIM e em 2019 registrou 69\% se aproximando assim da última etapa da Curva de Inovação de Roger.

\section{Considerações Finais}

A trajetória de implementação do BIM no Reino Unido evidência a importância de se conciliar outras técnicas e conceitos como o Lean Construction. Conforma afirmou Dave et al. (2013), que o ambiente do Lean Construction, com ênfase na previsibilidade e disciplina, mas também na colaboração e na experimentação, facilitaria a introdução e implementação de tecnologias baseadas em BIM e aumentaria sua eficácia. Sendo especialmente relevante durante a adoção da estratégica inicial de uma nova tecnologia aplicada no ciclo de vida do projeto.

Enquanto o Reino Unido optou por uma adoção gradativa do BIM o Brasil demonstra em sua estratégia a urgência em alcançar o nível 2 de maturidade e para isso estabeleceu um período curto para a sua adoção.

Esta urgência do Brasil se dá por conta de alguns objetivos estabelecidos no BIM BR. São eles: precisão no planejamento de execução de obras proporcionando maior confiabilidade de cronogramas e orçamentação, redução dos prazos para conclusão de obras; melhoria da transparência nos processos licitatórios; redução da necessidade de aditivos contratuais de alteração do projeto, de elevação de valor e de prorrogação de prazo de conclusão e de entrega da obra.

Ainda se faz importante destacar que o BIM não é uma ferramenta ou software a ser adotado, e sim um processo que requer mudanças organizacional e cultural da indústria da construção como é explicado por Dave et al., (2013). E, portanto, não se pode deixar de lado o fator humano nesta transição de um processo pouco informatizado para um totalmente informatização.

Portanto seria viável a inclusão da metodologia BIM na grade curricular dos cursos de engenharia civil e arquitetura de forma que de médio a longo prazo os profissionais a serem inseridos no mercado de trabalho já estejam familiarizados com o BIM.

Rogers (1983) afirma que adoção é um processo individual composto por estágios, diferente da difusão que é o 
fenômeno que sugere quando uma inovação é disseminada. Se a estratégia BIM BR precisa lograr sucesso na sua adoção de forma que a sua disseminação não seja comprometida. Contudo, como o processo de adoção do BIM no Brasil se encontra no início é preciso aguardar para verificar a eficiência da estratégia adotada pelo governo.

Infelizmente a estratégia BIM BR não deixa claro como se dará o acompanhamento da implementação e análise dos resultados obtidos de forma que possibilite a verificação das metas estabelecidas pelo governo. Tomioka e Neves (2020) demonstram que é necessário avaliar um conjunto de indicadores para analisar o desempenho das inovações na construção civil, porque o sistema tradicional não é adequado. Uma opção seria o Ease of Intergration Index (EOII) apresentado por Fenby-Taylor, H., et al. (2016), um índice avalia indicadores de economia, política e governança para analisar a facilidade de integração de um determinado país ao BIM.

Para estudo futuro sugere-se discutir a viabilidade do EOII como índice de verificação das metas estabelecidas pelo governo brasileiro, pois é necessário avaliar se ele é aplicável ao cenário brasileiro. Se esses indicadores utilizados no cálculo do EOII conseguem representar todos os segmentos a serem beneficiados pela implementação do BIM. E analisar se não seria o caso de uma adaptação ou até mesmo a elaboração de um indicador de desempenho. As respostas para esses questionamentos devem ser alcançadas por meio pesquisas futuras.

\section{Referências}

Bryde, D., Broquetas, M., \& Volm, J. M. (2013). The project benefits of building information modelling (BIM). International Journal of Project Management, 31(7), 971-980.

Bulgacov, S. (1998). Estudos comparativo e de caso de organizações de estratégias. Organizações \& Sociedade, 5(11), 53-76.

Center for Digital Built Britain (2018). Year One Report: Towards a digital built Britain. Department for Business Energy \& Industrial Strategy, CDBB Centre for Digital Built Britain.

Dave, B., et al. (2013). Implementing lean in construction: Lean construction and BIM-CIRIA Guide C725.

Eastman, C. M., et al. (2011). BIM handbook: A guide to building information modeling for owners, managers, designers, engineers, and contractors. John Wiley \& Sons, 485

Encontro Técnico Nacional de Auditoria de Obras Públicas (2019). Utilização da Plataforma BIM em Obras Públicas. IBRAOP- Instituto Brasileiro de Auditoria de Obras Públicas.

Fenby-Taylor, H., et al. (2016). Scotland Global BIM Study: Scottish Futures Trusts, 17.

Green, S. D., \& May, S. C. (2005). Lean construction: arenas of enactment, models of diffusion and the meaning of 'leanness'. Building research \& information, 33(6), 498-511.

HM Government (2011a). Government Construction Strategy. Cabinet Office.

HM Government. (2011b). BIM Management for Value, Cost \& Carbon Improvement; A Report for the Government Construction Client Group, Building Information Modelling (BIM) Working Party Strategy Paper.

HM Government. (2016). Secretary of State for International Trade signs building agreement with Brazil. 2016. https://www.gov.uk/business-and-industry.

McAuley, B., Hore, A., \& West, R. (2017). BICP global BIM study-Lessons for Ireland's BIM programme.

Khanzode, A., Fischer, M., Reed, D., \& Ballard, G. (2006). A guide to applying the principles of virtual design \& construction (VDC) to the lean project delivery process. CIFE, Stanford University, Palo Alto, CA.

Koskela, L. (1992). Application of The New Production Philosophy to Construction (v 72). Stanford: Stanford University.

Construction, M. H. (2014). The business value of BIM for construction in major global markets: How contractors around the world are driving innovation with building information modeling. Smart MarketReport, 1-60.

Miettinen, R., \& Paavola, S. (2014). Beyond the BIM utopia: Approaches to the development and implementation of building information modeling. Automation in Construction, 43, 84-91.

Inteligente, B. B. C. (2018). Brasil: Ministério da Indústria. Comércio Exterior e Serviços.

Moore, F. (2018). Digital Delivery and Structured Information Management: BIM level 2 Programme Management. CDBB.

National Building Specification (NBS). (2016). NBS National BIM Report 2016. 
Research, Society and Development, v. 10, n. 1, e25310111686, 2021

(CC BY 4.0) | ISSN 2525-3409 | DOI: http://dx.doi.org/10.33448/rsd-v10i1.11686

National Building Specification (NBS). (2019). National BIM report 2019-the definitive industry update.

National Building Specification (NBS). (2015). National BIM report 2015. Royal Institute of British Architects.

Pereira, A. S., et al. (2018). Metodologia da pesquisa científica. UAB/NTE/UFSM. https://repositorio.ufsm.br/bitstream/handle/1/15824/Lic_Computacao_Metodologia-Pesquisa-Cientifica.pdf?sequence=1.

Rischmoller, L., Alarcón, L. F., \& Koskela, L. (2006). Improving value generation in the design process of industrial projects using CAVT. Journal of Management in Engineering, 22(2), 52-60.

Rogers, E. M. (2010). Diffusion of innovations. Simon and Schuster.

Sacks, R., Dave, B., Koskela, L., \& Owen, R. (2009). Analysis framework for the interaction between lean construction and building information modelling.

Sacks, R., Koskela, L., Dave, B. A., \& Owen, R. (2010). Interaction of lean and building information modeling in construction. Journal of Construction Engineering and Management, 136(9), 968-980.

Shane, J. S., Molenaar, K. R., Anderson, S., \& Schexnayder, C. (2009). Construction project cost escalation factors. Journal of Management in Engineering, 25(4), 221-229.

Smith, P. (2014a). BIM \& the 5D project cost manager. Selected Papers From The 27th Ipma (International Project Management Association).

Smith, P. (2014b). BIM implementation-global strategies. Procedia Engineering, 85, 482-492.

Tomioka, A. M. \& Neves, J. M. S. das. (2019). Aplicação do Lean Construction e Agile Methods na Gestão de Obras Civis: Uma Análise Bibliométrica. Revista Eletrônica de Tecnologia e Cultura, Jundiaí. http://www.fatecjd.edu.br/portal/wp-content/uploads/2019/10/RETC-24-outubro-de-2019.pdf. 39-50.

Tomioka, A. M., \& Neves, J. M. S. das. (2020). Indicadores de desempenho para melhorar as dimensões competitivas da empresa de construção. Research, Society and Development, 9(5), e54953130. https://doi.org/10.33448/rsd-v9i5.3130

Tribunal de Contas da União (TCU). (2018). Diagnóstico das Obras Paralisadas: Identificação das Principais Causas e das Oportunidades de Melhoria, Recomendações e Monitoramento.

Tribunal de Contas da União (TCU). (2006). Levantamento de Auditoria: Diagnóstico sobre as Obras Inacabadas Realizadas com Recursos da União Verificação de Oportunidades para Aperfeiçoamento de Normativos e da Sistemática de Controle das Obras Públicas, Determinações, Recomendações, Sugestões, Ciência e Arquivamento.

Womack, J. P., \& Jones, D. T. (2004). A máquina que mudou o mundo. Gulf Professional Publishing. 